\title{
Wiener Index of Directed and Weighted Graphs by MATLAB Program
}

\author{
K. Thilakam ${ }^{1}$, A. Sumathi ${ }^{2}$ \\ ${ }^{1,} P G$ and Research Department of Mathematics, Seethalakshmi Ramaswami College, Tiruchirappalli-2 \\ ${ }^{2,}$ Department of Mathematics, Seethalakshmi Ramaswami College, Tiruchirappalli-2
}

\begin{abstract}
The Wiener index is the one of the oldest and most commonlyused topological indices in the quantitative structure-property relationships. It is defined by the sum of the distances between all (ordered) pairs of vertices of $G$. In this paper, we use MATLAB program for finding the Wiener index of the vertex weighted, edge weighted directed and undirected graphs
\end{abstract}

Keywords: Distance Sum, MATLAB, Sparse Matrix, Wiener Index

\section{Introduction}

The Wiener index $\mathbf{W}(\mathbf{G})$ is a distance-based topological invariant, it much used in the study of the structure-property and the structure-activity relationships of several classes of biochemically interesting compounds introduced by Harold Wiener in 1947 for predicting boiling points $(b . p)$ of alkanes based on the formula $\boldsymbol{b} \cdot \boldsymbol{p}=\alpha W+\beta w(3)+\gamma$, where $\alpha, \beta, \gamma$ are empirical constants, and $\mathbf{w}(3)$ is called path number. It was the first application of the Wiener index in 1947[11, 12]. In a sequence of the papers published in the following years, he pointed out the versatility of his index in structure property investigations. It is defined as the half sum of the distances between all pairs of vertices of $\mathrm{G} . W(G)=\frac{1}{2} \sum_{u, v \in G} d(u, v)$. Various algorithms for the calculation of the Wiener index have been proposed in the chemical literature[5,6,7,8].In this paper, we find $\mathrm{W}(\mathrm{G})$ in minimum timewith the help of MATLAB through Adjacency matrix of a graph and existing formula of Wiener index . Especially it is convenient for the calculation of $\mathrm{W}$ of moderately large molecule. The Adjacency matrix of a graph $G$ with $n$ vertices and no parallel edges is an $n$ by $n$ matrix $A(G)$ defined as

$$
\begin{aligned}
\mathrm{a}_{\mathrm{ij}} & =1, & & \text { if there is an edgebetweenith and jth vertices and } \\
& =0, & & \text { if there is no edge between them }
\end{aligned}
$$

Notation:[11, 12, 13]

$$
W(G)=\frac{1}{2} \sum_{u, v \in G} d(u, v)=\sum_{u<v} d(u, v)=\sum_{i<j} d\left(u_{i}, u_{j}\right)
$$

Let $d(u, v)$ denote the length of a shortest path in $G$ between u and v. If no such path exists, we defined $(u, v)=\infty$. Generally, the Floyd-Warshall algorithm and Johnson's algorithm are the most well-known all pair shortest path algorithms. In recent back, we have discussed the $\mathrm{W}(\mathrm{G})$ of undirected unweighted graph[10] In this paper,we have determined Wiener index of aweighted graph and directed graph using Johnson's algorithmthrough MATLAB.In MATLAB, N-by-N sparse matrix represents a graph. Where, sparse matrix is a matrix in which most of the elements are zero. By contrast, if most of the elements are nonzero, then the matrix is considered dense. The fraction of zero elements (non-zero elements) in a matrix is called the sparsity (density).Nonzero entries in matrix $G$ represent the weights of the edges. By default, it gets weight of the edges for undirected graph as one. Johnson's algorithm has a time complexity of $\mathrm{O}(\mathrm{N} * \log (\mathrm{N})+\mathrm{N} * \mathrm{E})$, where $\mathrm{N}$ and $\mathrm{E}$ are the number of nodes and edges respectively.

\section{Algorithm For Finding Wiener Index Of Vertex Or Edge Weighted Directed / Undirected Graph}

Let $\mathrm{G}$ be a given connected graph with $\mathrm{n}$ vertices.

Input: Adjacency list and Weights of vertices (edges) of a directed (undirected) graph G

Step 1: Determine sparse matrix G

Step 2: Determine Distance Matrix D of G

Step 3: Find Wiener index $\mathrm{W}$ of $\mathrm{G}$

Output: Resulting Graph G and its Wiener Index 


\section{Finding The Wiener Index Of Weighted Undirectedgraph}

\subsection{Wiener Index of Vertex-Weighted Graphs}

A vertex-weighted graph $(\mathrm{G}, \mathrm{w})$ is a graph $\mathrm{G}$ together with a function $\mathrm{w}: \mathrm{V}(\mathrm{G}) \rightarrow \mathrm{N}^{+}$.(Evidently, we could have chosen for vertex-weights (positive) real numbers. For the present considerations, however, weighting of the vertices with positive integers will suffice.) From now on vertex-weighted graphs in which all weights are positive integers will be called simply weighted graphs. The Wiener number $W(G, w)$ of a weighted graph $(\mathrm{G}, \mathrm{w})$ is defined as

$$
W(G, w)=\frac{1}{2} \sum_{u, v \in V(G)} w(u) w(v) d_{G}(u, v)
$$

Note that if $w(u)=1$ holds for all vertices $u \in V(G)$, then $W(G, w)=W(G)$. More generally, if $w$ is a constant function, say $\mathrm{w} \equiv \mathrm{m}$, then $\mathrm{W}(\mathrm{G}, \mathrm{w})=\mathrm{m}^{2} \mathrm{~W}(\mathrm{G})$. Observe also that if the distance matrix of a graph $\mathrm{G}$ is given, then it is no more difficult to compute $W(\mathrm{G}, \mathrm{w})$ than $\mathrm{W}(\mathrm{G})$. $[2,9]$

The following Example illustrates finding $\mathrm{W}$ of weighted cycle graph through $\mathrm{A}(\mathrm{G})$ and $\mathrm{w}\left(\mathrm{u}_{\mathrm{i}}\right), \mathrm{i}=1$ to $\mathrm{n}$.

\section{Numerical Example:1}

clc

clearall

$\mathrm{n}=\operatorname{input}($ 'Cycle with vertices $\mathrm{n}=$ ');

$\mathrm{M}=$ input('weights of the vertices in matrix form=');

$\mathrm{A}=[\mathrm{]}$;

for $\mathrm{i}=1: \mathrm{n}-1$

Define an Empty matrix A

$\mathrm{A}(\mathrm{i}, \mathrm{i}+1)=1 ; \mathrm{A}(\mathrm{i}+1, \mathrm{i})=1$

$\mathrm{A}(1, \mathrm{n})=1 ; \mathrm{A}(\mathrm{n}, 1)=1$;

end

To determine Adjacency matrix A of G

$\mathrm{G}=\operatorname{sparse}(\mathrm{A})$;

$\mathrm{D}=$ graphallshortestpaths $(\mathrm{G}$, 'directed',false $)$

Distance matrix $\mathrm{D}$ of $\mathrm{G}$

$\mathrm{W}=\left(\left(\mathrm{M}^{*} \mathrm{D}\right) * \mathrm{M}^{\prime}\right) / 2$

fprintf('Wiener index of $\mathrm{Cn}, \mathrm{W}=\% \mathrm{~d} \backslash \mathrm{n}$ ', $\mathrm{W})$;

Wiener index $W$ of $G$

Wiener indices of weighted graphs, as defined above, seem not to be previously studied (in either mathematical or chemical literature). Exceptionally, one of the present authors did some work [4] on W(G,w), where the weight $\mathrm{w}(\mathrm{u})$ was set to be equal to the degree of the vertex $u$.

The following Example illustrates finding W of weighted cycle graph through $A(G)$ and defined $w\left(u_{i}\right), i=1$ to $n$.

\section{Numerical Example:2}

clc

clearall

$\mathrm{n}=$ input('Cycle with vertices $\mathrm{n}=$ ');

$\mathrm{A}=[\mathrm{]}$;

for $\mathrm{i}=1: \mathrm{n}-1$

$\mathrm{A}(\mathrm{i}, \mathrm{i}+1)=1 ; \mathrm{A}(\mathrm{i}+1, \mathrm{i})=1$;

$\mathrm{A}(1, \mathrm{n})=1 ; \mathrm{A}(\mathrm{n}, 1)=1$;

end

A;

$\mathrm{G}=\operatorname{sparse}(\mathrm{A})$;

$\mathrm{D}=$ graphallshortestpaths $(\mathrm{G}$, 'directed',false $)$

$\mathrm{M}=\operatorname{sum}(\mathrm{A}, 1)$

$\mathrm{W}=\left(\left(\mathrm{M}^{*} \mathrm{D}\right) * \mathrm{M}^{\prime}\right) / 2$

$\mathrm{M}=$ degree of $\mathrm{u}_{\mathrm{i}}$ in matrix form

fprintf('Wiener index of $\mathrm{Cn}, \mathrm{W}=\% \mathrm{~d} \backslash \mathrm{n}$ ' , W); 


\section{Execution of the Example2}

For instance, the following is the execution of simple MATLAB Program in the command window for the vertex weighted cycleundirected graph with $n=10$

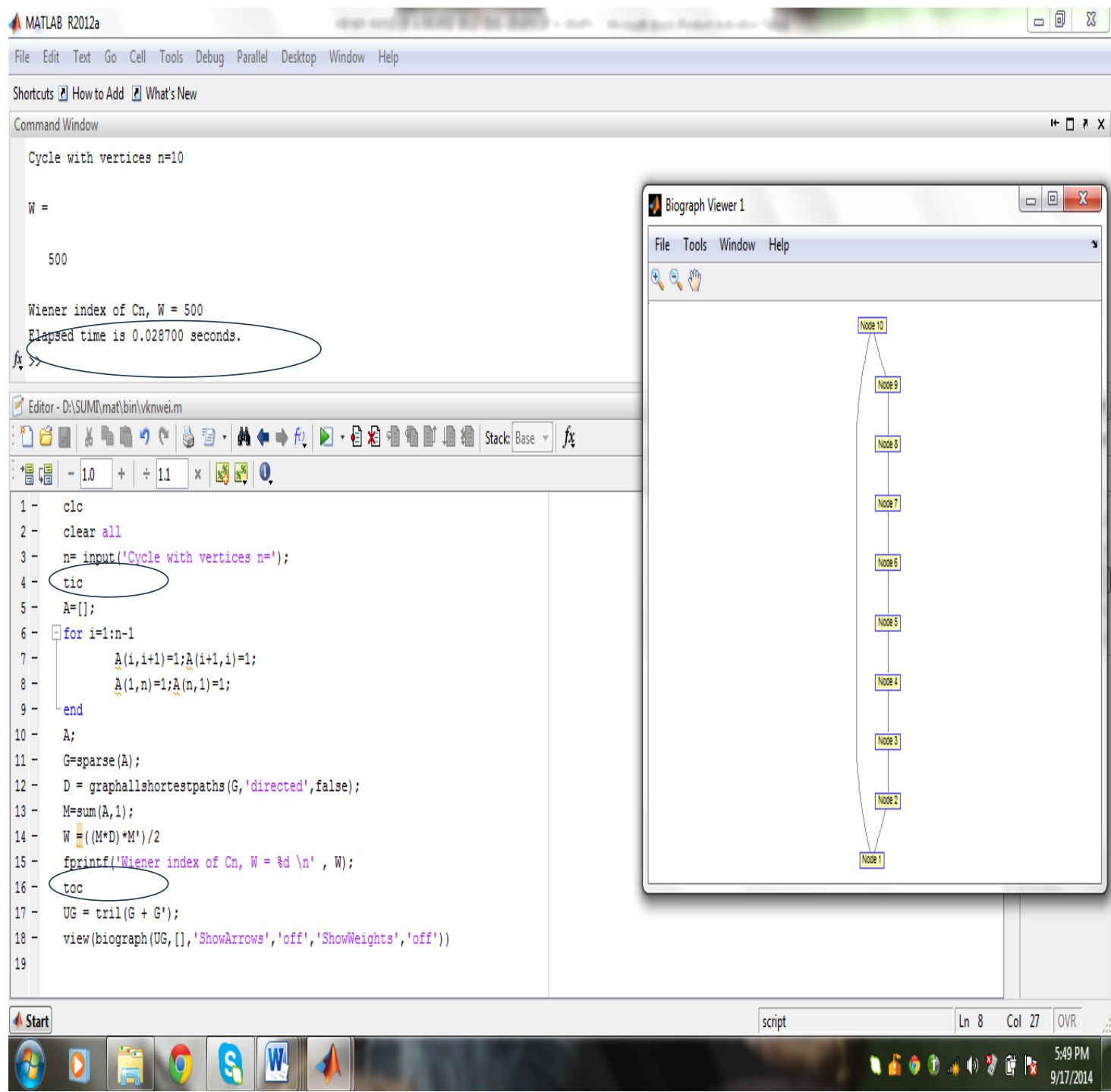

Note that in MATLAB,toc syntax stops a stopwatch timer started by the tic function, and displays the time elapsed in seconds.toc(ticID) syntax displays the time elapsed since the tic command corresponding to ticID. elapsedTime $=$ toc syntax stores the elapsed time in a variable. elapsedTime $=$ toc(ticID) syntax stores in a variable the time elapsed since the tic command corresponding to ticID.Theoutputargumentelapsed time is the Scalar double, it represents the time elapsed between tic and toc commands, in seconds. [1, 14]

\subsection{Wiener Index of Edge-Weighted Undirected Graph}

In MATLAB, $D=$ graphallshortestpaths $(G)$ finds the shortest paths between every pair of nodes in the graph represented by matrix $G$, using Johnson's algorithm. Nonzero entries in matrix G represent the weights of the edges. Elements in the diagonal of this matrix are always 0, indicating the source node and target node are the same. A 0 not in the diagonal indicates that the distance between the source node and target node is 0 . An Inf indicates there is no path between the source node and the target node.

\section{Finding The Wiener Index Of Un Weighted/Weighted Directed Graph}

A directed graph Gis given by a set of vertices $V=V(G)$ and a set of ordered pairs of vertices $E=E(G)$ called directed edges or arcs. The number of vertices of Gis denoted by nand the number of arcs is denoted by $m$. A (directed) path in $G$ is a sequence of vertices $v_{0}, v_{1}, \ldots . . v_{n}$ such that $v_{i-1} v_{i}$ is an arc of Gfor all i. The distance $d(u, v)$ is the length of a shortest path from uto $v$. In digraphs, in general $d(u, v))=d(v, u)$ does not hold. [3] 
The following program illustrates finding $\mathrm{W}$ of directed graph

\section{Program:4.1}

clc

clearall

$\mathrm{n}=$ input('Graph with vertices $\mathrm{n}=$ ');

$\mathrm{A}=[\mathrm{]}$;

for

......

$\cdots \cdots$

end

A;

DG=sparse(A);

$\mathrm{DM}=$ graphallshortestpaths(DG,'directed',true)

$\mathrm{W}=\operatorname{sum}(\operatorname{sum}(\mathrm{DM}))$;

fprintf('Wiener index of a Graph, W =\%d In', W);

Define an Empty matrix A

To determine Adjacency matrix Aof directed graph $\mathrm{G}$

'directed', true represents

Wiener index $\mathrm{W}$ of $\mathrm{G}$ view(biograph(DG,[],'ShowArrows','on','ShowWeights','off'))

\section{Execution of the Numerical Example 4.2}

For instance, the following is the execution of simple MATLAB Program in the command window forthe edge weighted cycle digraph with $n=4$. The program asks the user to enter the number of vertices of $G$. Nonzero entries in the matrix of directed graph $G$ represent the weights of the edges. Therefore we define the Adjacency matrix of some particular digraph $\mathrm{G}$ with weights.

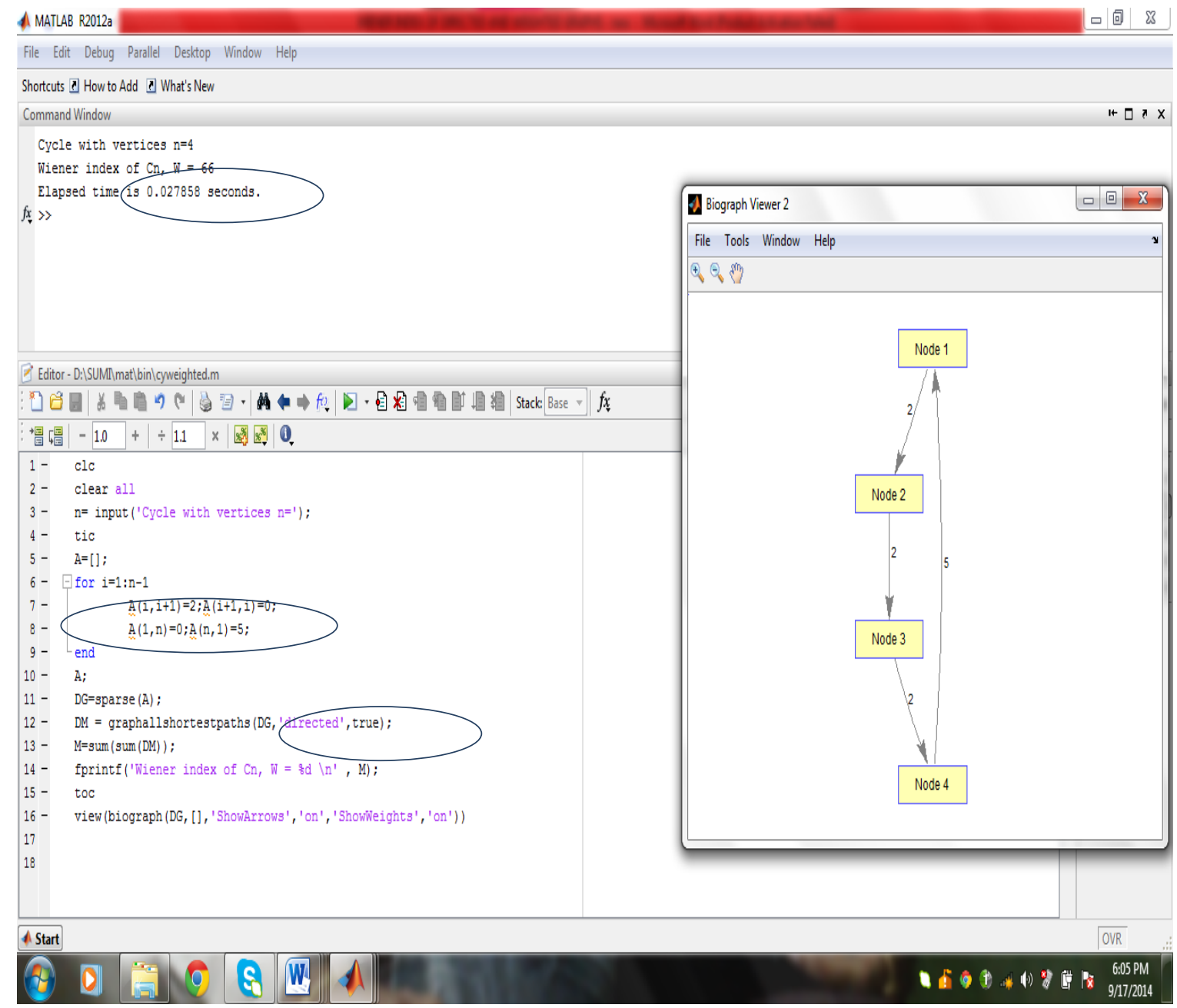




\section{Conclusion}

With the help of above MATLAB program, we can easily find out the Wiener index of vertex weighted, edge weighted undirected and directed molecular graphs with large molecular size in shortest time.

\section{References}

[1] Amos Gilat, "MATLAB An Introduction with Applications”, John Wiley \& Sons, Inc. U.K, 2004.

[2] Bla`Zmazek and JanezZerovnik,Computing the Weighted Wiener and Szeged Number on Weighted Cactus Graphs in Linear Time, Croaticachemicaacta, CAACAA 76 (2) 137-143(2003) ISSN-0011-1643

[3] Igor Pesek, MajaRotovnik, DamirVuki cevi'candJanez 'Zerovnik, Wiener Number of Directed Graphs and Its Relation to the Oriented Network Design Problem, MATCH Commun. Math. Comput. Chem. 64 (2010) 727-742,ISSN 0340 - 6253

[4] Ivan Gutman, Selected properties of the Schultz molecular topological index, J. Chem. Inf. Comput. Sci. 34 (1994) 1087-1089

[5] Mohar.B, and Pisanski.T, How to compute the Wiener index of a graph, Journal of mathematical Chemistry, 2 (1988) 267-277.

[6] Muller. W. R, Szymanski. K., and Knop. J. V, “An Algorithm for construction of the Molecular Distance Matrix”,Journal of Computational Chemistry, 8, No.2, (1987), 170-173.

[7] Nikolic .S, Trianjstic .N and Mihalic .Z, The Wiener Index: Developments and applications, Croat. Chem.Acta., 68(1995) 105129.

[8] Rama Krishnan .S, Senbagamalar .J, BaskerBabujee .J,International Journal of Computing Algorithm,Vol:02,Oct 2013,PP:224234

[9] Sandi Klav`zar, Ivan Gutman, Wiener number of vertex-weighted graphs and a chemical application

[10] Thilakam K, Sumathi A, How to Compute the Wiener index of a graph using MATLAB,International Journal of Applied Mathematics\& Statistical Sciences, ISSN: 2319-3972; Vol.2, Issue 5, Nov 2013, 143-148.

[11] Wiener H, J. Chem. Phys. 15 (1947) 766.

[12] Wiener H, Structural determination of paraffin boiling points, J. Amer. Chem. Soc.69 (1947) 17-20.

[13] http://en.wikipedia.org/wiki/moleculargraph

[14] http://www.mathworks.in/products/matlab 